\title{
Rapid Urease Test and Faecal Antigen Detection for Rapid Diagnosis of Helicobacter pylori Infection in Dyspepsia
}

\author{
Hasan Sedeek Mahmoud1, Hanan M. Fayed2, Ghada S. Osman ${ }^{3}$ \\ ${ }^{1}$ Tropical Medicine and Gastroenterology Department, Qena Faculty of Medicine, South Valley University, \\ Qena, Egypt \\ ${ }^{2}$ Department of Clinical Pathology, Qena Faculty of Medicine, South Valley University, Qena, Egypt \\ ${ }^{3}$ Department of Pathology, Qena Faculty of Medicine, South Valley University, Qena, Egypt \\ Email: hasan_sedeek@yahoo.com
}

Received 31 December 2015; accepted 20 January 2016; published 25 January 2016

Copyright (C) 2016 by authors and Scientific Research Publishing Inc.

This work is licensed under the Creative Commons Attribution International License (CC BY).

http://creativecommons.org/licenses/by/4.0/

(c) (i) Open Access

\begin{abstract}
Background: Helicobacter pylori $(H$. pylori) is considered as one of the most prevalent gastric infections which cause chronic gastritis and predispose to cancer stomach. So, diagnosis and eradication should be rapid to decrease the risk of gastric cancer. Aim of the study: To evaluate the role of rapid urease test (RUT) and faecal antigen test (FAT) added to serological test for rapid diagnosis of active $H$. pylori infection. Patients and methods: 270 patients with dyspepsia and positive serology for $H$. pylori infection were included. Two antral and two corporal gastric biopsies were taken for RUT and Histopathological examination. Fresh stool samples were obtained from all patients for FAT. Results: The mean age of the studied patients was $45 \pm 25$. $H$. pylori infection was found in $256(94.8 \%)$ of the included patients: $236(92.18 \%)$ with positive all tests, $5(1.95 \%)$ with positive both RUT and FAT, 8 (3.12\%) with positive both histology and RUT and 7 (2.73\%) with positive histology and FAT. The sensitivity, specificity and positive predictive values for RUT were as follows: $\mathbf{9 7 . 2 7 \%}, \mathbf{8 5 . 7 1 \%}$ and $\mathbf{9 9 . 2 0 \%}$ respectively and $96.88 \%, 85.71 \%$ and $99.20 \%$ respectively for FAT. Conclusions: RUT or FAT in patients with positive serological test could be used for rapid diagnosis of active $H$. pylori infection with good sensitivity and specificity without waiting for the results of histology or culture.
\end{abstract}

\section{Keywords}

Rapid Urease Test, Faecal Antigen Test, Helicobacter Pylori

\section{Introduction}

The prevalence of $H$. pylori infection varies strongly worldwide, with less than $40 \%$ prevalence in developed 
countries and more than $80 \%$ - 90\% in developing countries [1] [2].

Worldwide the most common cause of chronic gastritis is infection with $H$. pylori. It causes progressive damage to the gastric mucosa and is now accepted as playing a causative role in a number of important diseases, including duodenal ulcer disease, gastric ulcer disease, gastric adenocarcinoma and gastric mucosa-associated lymphoid tissue (MALT) lymphoma [3] [4], and involved in the pathogenesis of idiopathic thrombocytopenic purpura, iron deficiency anemia and vitamin B12 deficiency [5].

Several diagnostic methods for $H$. pylori infection are available, either invasive, which require endoscopy and gastric biopsies (rapid urease test, histology and cultures) or non-invasive (urea C13 breath test, stool antigen detection and serology), all of them exhibiting both advantages and disadvantages regarding availability, rapidity of results, value, and diagnostic accuracy [6] [7].

No H. pylori diagnostic method has $100 \%$ sensitivity [8]. Hence, unless a combination of test methods is used, misdiagnosis is more likely and this may well explain some of the negative $H$. pylori duodenal ulcers that appear otherwise to be idiopathic. Use of two or more tests together increases the sensitivity and the negative predictive value. However, even if two diagnostic tests are negative, a third may be needed in certain circumstances such as in the presence of widespread atrophy or intestinal metaplasia [9], as $H$. pylori tends to disappear naturally from the gastric mucosa with the expansion of these histological lesions [10].

In the current study we aimed to evaluate the role of RUT and FAT added to serological test for rapid diagnosis of active $H$. pylori infection.

\section{Patients and Methods}

\subsection{Inclusion Criteria}

Patients presented by dyspeptic symptoms (epigastric pain or burning sensation, nausea, vomiting, heartburn, bloating, early satiety) with positive serological antibody test for $H$. pylori, were included from the out-patient gastroenterology clinic of Qena university hospital from April 2014 to April 2015; they were not previously screened or treated for H. pylori infection.

\subsection{Study Design}

The study design is the prospective cross-sectional study.

\subsection{Exclusion Criteria}

Patients, who had a history of PPI, H2 receptor antagonist, bismuth, warfarin, fluoxetin, or steroid use within two weeks, or antibiotic use within four weeks as well as those with severe medical illness, active gastrointestinal bleeding, and history of gastric surgery and $H$. pylori eradication, were excluded from the study.

\subsection{Methods}

All included patients underwent upper endoscopy using Olympus, GIF-XQ260 instrument, and we obtained two biopsy specimens from the antrum and two biopsies from the corpus of the stomach. One biopsy from each area was used for RUT and the others were formalin fixed, stained with modified Giemsa, and examined for the presence of $H$. pylori by a pathologist. The RUT was performed using Pronto Dry kit (Gastrex Sarl, 21640 Gilly les Citeaux, France). First we peel back the label of the kit thus exposing the dot. With sterile $19 \mathrm{G}$ needle we remove the specimen from the biopsy forceps and place them into the Pronto Dry then we accurate re-seal the pronto dry and press the label over the dot with fingers to squeeze the tissue juice out of the specimen and read within one hour for all cases. The test was considered positive if the external ring area was deepening in color and expanding in size. The internal dot always stays yellow. If urease is present in the tissue, an expanding magenta color external ring will be noted around the biopsy specimen, or the Pronto Dry will gradually change to a deep orange, then to magenta color. But negative result is when the external ring is still yellow 1 hour after insertion of the specimen. For $H$. pylori antigen detection, fresh stool sample for each patient was sent for laboratory assessment. Ridascreen FemtoLab H. pylori test (R-Biopharm AG, Darmstadt, Germany) was performed according to the manufacturer's rules. This test was done using dual amplification technology and coating with a monoclonal antibody directed against the catalase of H. pylori. After the color change at the end of the test, the 
intensity was determined spectrophotometrically with a wavelength of $450 \mathrm{~nm}$. Absorbance was expressed as an optical density (OD) value. In accordance with the manufacturer's guidelines; an OD of $<0.150$ was defined as a negative test result and an $\mathrm{OD}$ of $\geq 0.150$ was defined as a positive test result.

Patient is considered positive if showed positive serology and positive at least two other tests; histopathology, faecal antigen and RUT.

\subsection{Ethical Consideration}

Informed consent from included patients and approval from the faculty research ethical committee were obtained before proceeding.

\subsection{Statistical Analysis}

Data entry and analysis were done using statistical package of social science (SPSS) version 20. The data arepresented as means \pm SD and number, (\%). calculation of sensitivity, specificity, positive predictive and negative predictive values were online calculated: https://www.medcalc.org/calc/diagnostic test.php.

Sensitivity $=\mathrm{a} / \mathrm{a}+\mathrm{b}$, specificity $=\mathrm{d} / \mathrm{c}+\mathrm{d}, \overrightarrow{P P V}=\mathrm{a} / \mathrm{a}+\mathrm{c}$ and NPV $=\mathrm{d} / \mathrm{b}+\mathrm{d} ; \mathrm{a}=$ true positive, $\mathrm{b}=$ false negative, $\mathrm{c}=$ false positive and $\mathrm{d}=$ true negative.

\section{Results}

The current study included 270 patients with their mean ages were $45 \pm 25.150$ (55.6\%) of them were males and 120 (44.4\%) were females (Table 1).

H. pylori infection was found in 256 (94.8\%) of the included patients and they were as follow; 236 (92.18\%) with positive all tests (histology, RUT, FAT), 5 (1.95\%) with positive RUT and FAT, 8 (3.12\%) with positive histology and RUT and 7 (2.73\%) with positive histology and FAT (Table 2).

Table 1. Demographic data for all patients.

\begin{tabular}{cc}
\hline Parameter & Number (\%) \\
Total & 270 \\
Age & $45 \pm 25$ \\
Sex & $150(55.6 \%)$ \\
Male & $120(44.4 \%)$ \\
Female & \\
Residence & $190(70.4 \%)$ \\
Rural & $80(29.6 \%)$ \\
Urban &
\end{tabular}

Data expressed number (\%) or mean \pm SD.

Table 2. State of $H$. pylori infection in all tests.

\begin{tabular}{cccccc}
\hline \multirow{2}{*}{ Number } & \multicolumn{5}{c}{ Test type and result } \\
\cline { 2 - 6 } & Serology $(\mathrm{Ab})$ & Histology & RUT & Faecal Ag & *State of $H$. pylori \\
\hline 236 & + & + & + & + & + \\
5 & + & - & + & + & + \\
8 & + & + & + & + & + \\
2 & + & - & - & + & + \\
7 & + & + & - & - & - \\
2 & + & - & + & - & - \\
\hline
\end{tabular}

*State of $H$. pylori either there is active infection (+) or, no active infection (-). 
The probability of detection of $H$. pylori infection by FAT and RUT was high and were comparable to each other. The sensitivity, specificity, positive predictive value (PPV) and negative predictive value (NPV) were as follow: $97.27 \%, 85.71 \%, 99.20 \%$ and $63.16 \%$ respectively for RUT and $96.88 \%, 85.71 \%, 99.20 \%$ and $60 \%$ respectively for FAT, and those for histology were as follow: $98.05 \%, 100 \%, 100 \%$ and $73.68 \%$ respectively (Table 3) (Figure 1).

\section{Discussion}

Helicobacter pylori is the cause of gastritis and most cases of peptic ulcer disease (PUD) and its long term infection predispose to multiple diseases including: gastric adenocarcinoma; six-foldincrease and it is considered as a class I carcinogen [11], gastric mucosa associated lymphoid tissue lymphomas, short stature in young girls, development of vasospastic disorders and possibly skin immunopathology such as urticaria. Early diagnosis and eradication of this pathogen could protect the human from these dangerous diseases.

Cure of $H$. pylori infection leads to healing of the inflamed gastric mucosa, which may return to normal. $H$. pylori eradication may improve or resolve dyspeptic symptoms and usually cures PUD. H. pylori gastritis is a disease which can be cured and thus prevent severe complications. If $H$. pylori gastritis has progressed to more severe forms of gastritis, including atrophic gastritis with or without intestinal metaplasia, or severe corpus predominant gastritis, the risk of gastric cancer is increased and eradication of the infection at this stage needs to be integrated with a follow-up strategy [4] [12]-[14].

Because faecal antigen detection is rapid, technically simple, and ease of sample collection and the RUT is a rapid, cheap and simple test we aimed to compare these two rapid tests for diagnosis of $H$. pylori infection.

In the current study RUT and FAT showed good specificity and sensitivity in patients with positive serology for detection of active $H$. pylori infection. Non-invasive diagnostic tests such as faecal antigen test, serological parameters and the [13C]-urea breath test serve as surrogate markers of $H$. pylori gastritis and indicators of gastritis severity [4].

Andrews and his colleague's compare three stool antigen tests for $H$. pylori detection and concluded that one of them may be considered as an alternative to urea breath testing in the initial diagnosis of patients with dyspepsia who do not require immediate endoscopy [15].

The RUT is an indirect test of the presence of $H$. pylori based on the presence of urease in or on the gastric mucosa. It has an advantage over serology in that it only detects the presence of an active infection. Different previous studies had evaluated RUT for diagnosis of $H$. pylori and reported that the sensitivity of RUT tests, as primary diagnostic tests, was high and has been reported to vary between approximately $80 \%$ and $100 \%$ and specificity between $97 \%$ and $99 \%$ [16] [17]. In order to save the patient money, some physicians have suggested taking biopsies for RUT as well as for histology from normal appearing mucosa and then, if the RUT is positive, discarding the histology specimens as unnecessary [18]-[20].

It is clear that prevention of infection with $H$. pylori could avoid chronic gastritis and its grave consequences

Table 3. Sensitivity, specificity, positive and negative predictive values for faecal stool antigen and rapid urease test.

\begin{tabular}{|c|c|c|c|c|}
\hline \multirow{2}{*}{ Test } & \multicolumn{3}{|c|}{ Parameter } & \multirow[b]{2}{*}{ NPV } \\
\hline & Sensitivity & Specificity & PPV & \\
\hline Faecal Ag & $96.88 \%$ & $85.71 \%$ & $99.20 \%$ & $60 \%$ \\
\hline RUT & $97.27 \%$ & $85.71 \%$ & $99.20 \%$ & $63.16 \%$ \\
\hline Histology & $98.05 \%$ & $100 \%$ & $100 \%$ & $73.68 \%$ \\
\hline
\end{tabular}

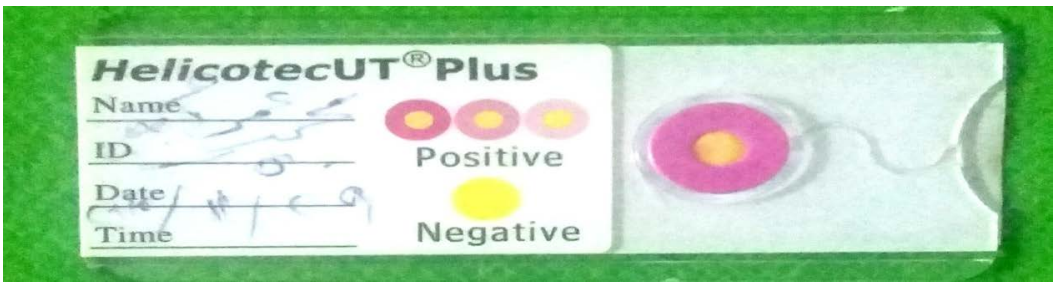

Figure 1. Rapid urease test showed positive result. 
especially gastric cancer. Based on the results of recent studies, the following measures could reduce transmission of $H$. pylori: Practice good hygiene and hand washing, especially with food preparation, all patients with chronic gastrointestinal symptoms that may be associated with $H$. pylori infection should be tested and treated to prevent exposure to family members, patients should complete the full course of therapy (antibiotics and acid blockers) to maximize the potential for cure, increase cruciferous vegetable intake (cauliflower, cabbage, and broccoli) and support policies to improve living conditions in developing countries [21]-[23].

\section{Conclusion}

RUT or FAT in patients with positive serological test could be used for rapid diagnosis of active $H$. pylori infection with good sensitivity and specificity without waiting for the results of histology or culture.

\section{Conflict of Interest}

Authors declared that no conflict of interest.

\section{References}

[1] Taylor, D.N. and Blaser, M.J. (1991) The Epidemiology of Helicobacter pylori Infection. Epidemiologic Reviews, 13, 42-59.

[2] Zhang, L., Blot, W.J., You, W.C., Chang, Y.S., Kneller, R.W., Jin, M.L., et al. (1996) Helicobacter pylori Antibodies in Relation to Precancerous Gastric Lesions in a High-Risk Chinese Population. Cancer Epidemiology, Biomarkers \& Prevention, 5, 627-630.

[3] Malfertheiner, P., Chan, F.K. and McColl, K.E. (2009) Peptic Ulcer Disease. Lancet, 374, 1449-1461. http://dx.doi.org/10.1016/S0140-6736(09)60938-7

[4] Malfertheiner, P., Mégraud, F., O’Morain, C.A., Atherton, J., Axon, A.T.R., Bazzoli, F., et al. (2012) Management of Helicobacter pylori Infection-The Maastricht IV Florence Consensus Report. Gut, 61, 646-664. http://dx.doi.org/10.1136/gutjnl-2012-302084

[5] Shiotani, A., Cen, P. and Graham, D.Y. (2013) Eradication of Gastric Cancer Is Now Both Possible and Practical. Seminars in Cancer Biology, 23, 492-501. http://dx.doi.org/10.1016/j.semcancer.2013.07.004

[6] Gisbert, J.P. (2000) Revisión crítica de los métodos diagnósticos de infección por Helicobacter pylori. Gastroenterología y Hepatología, 23, 135-143.

[7] De Boer, W.A., De Laat, L. and Megraud, F. (2000) Diagnosis of Helicobacter pylori Infection. Current Opinion in Gastroenterology, 16, S5-S10.

[8] Working Party of the European Helicobacter pylori Study Group (1997) Technical Annex: Tests Used to Assess Helicobacter pylori Infection. Gut, 41, S10-8.

[9] Quan, C. and Talley, N.J. (2002) Management of Peptic Ulcer Disease Not Related to Helicobacter pylori or NSAIDs. The American Journal of Gastroenterology, 97, 2950-2961. http://dx.doi.org/10.1111/j.1572-0241.2002.07068.x

[10] Craanen, M.E., Dekker, W., Blok, P., Ferwerda, J. and Tytgat, G.N. (1992) Intestinal Metaplasia and Helicobacter pylori: An Endoscopic Bioptic Study of the Gastric Antrum. Gut, 33, 16-20. http://dx.doi.org/10.1136/gut.33.1.16

[11] National Institute of Health Consensus Conference (1994) Helicobacter pylori in Peptic Ulcer Disease. JAMA, 272, 65-69.

[12] Asaka, M., Kato, M., Takahashi, S., Fukuda, Y., Sugiyama, T., Ota, H., et al. (2010) Guidelines for the Management of Helicobacter pylori Infection in Japan: 2009 Revised Edition. Helicobacter, 15, 1-20. http://dx.doi.org/10.1111/j.1523-5378.2009.00738.x

[13] Dinis-Ribeiro, M., Areia, M., de Vries, A.C., Marcos-Pinto, R., Monteiro-Soares, M., O’Connor, A., et al. (2012) Management of Precancerous Conditions and Lesions in the Stomach (MAPS): Guideline from the European Society of Gastrointestinal Endoscopy (ESGE), European Helicobacter Study Group (EHSG), European Society of Pathology (ESP) and the Sociedade Portuguesa de Endoscopia Digestiva (SPED). Endoscopy, 44, 74-94. http://dx.doi.org/10.1055/s-0031-1291491

[14] Naylor, G.M., Gotoda, T., Dixon, M., et al. (2006) Why Does Japan Have a High Incidence of Gastric Cancer? Comparison of Gastritis between UK and Japanese Patients. Gut, 55, 1545-1552. http://dx.doi.org/10.1136/gut.2005.080358

[15] Andrews, J., Marsden, B., Brown, D., Wong, V.S., Wood, E. and Kelsey, M. (2003) Comparison of Three Stool Antigen Tests for Helicobacter pylori Detection. Journal of Clinical Pathology, 56, 769-771. http://dx.doi.org/10.1136/jcp.56.10.769 
[16] Calvet, X., Sánchez-Delgado, J., Montserrat, A., Lario, S., Ramírez-Lázaro, M.J., Quesada, M., et al. (2009) Accuracy of Diagnostic Tests for Helicobacter pylori: A Reappraisal. Clinical Infectious Diseases, 48, 1385-1391. http://dx.doi.org/10.1086/598198

[17] Vaira, D. and Perna, F. (2007) How Useful Is the Rapid Urease Test for Evaluating the Success of Helicobacter pylori Eradication Therapy? Nature Reviews Gastroenterology and Hepatology, 4, 600-601. http://dx.doi.org/10.1038/ncpgasthep0966

[18] Perna, F., Ricci, C., Gatta, L., et al. (2005) Diagnostic Accuracy of a New Rapid Urease Test (Pronto Dry), before and after Treatment of Helicobacter pylori Infection. Minerva Gastroenterologica e Dietologica, 51, 247-254.

[19] Midolo, P. and Marshall, B.J. (2000) Accurate Diagnosis of Helicobacter pylori. Urease Tests. Gastroenterology Clinics of North America, 29, 871-878.

[20] Laine, L., Lewin, D., Naritoku, W., Estrada, R. and Cohen, H. (1996) Prospective Comparison of Commercially Available Rapid Urease Tests for the Diagnosis of Helicobacter pylori. Gastrointestinal Endoscopy, 44, 523-526. http://dx.doi.org/10.1016/S0016-5107(96)70002-0

[21] Cheung, T.K. and Wong, B.C. (2008) Treatment of Helicobacter pylori and Prevention of Gastric Cancer. Journal of Digestive Diseases, 9, 8-13.

[22] Wong, B.C., Lam, S.K., Wong, W.M., Chen, J.S., Zheng, T.T., Feng, R.E., Lai, K.C., Hu, W.H., Yuen, S.T., Leung, S.Y., Fong, D.Y., Ho, J., Ching, C.K. and Chen, J.S. (2004) Helicobacter pylori Eradication to Prevent Gastric Cancer in a High-Risk Region of China: A Randomized Controlled Trial. JAMA, 291, 187-194. http://dx.doi.org/10.1001/jama.291.2.187

[23] Kusters, J.G., van Vliet, A.H.M. and Kuipers, E.J. (2006) Pathogenesis of Helicobacter pylori Infection. Clinical Microbiology Reviews, 19, 449-490.

\section{Abbreviations:}

H. pylori: Helicobacter pylori;

RUT: rapid urease test;

FAT: faecal antigen test;

MALT: mucosa-associated lymphoid tissue;

OD: optical density;

PUD: peptic ulcer disease. 